\title{
LETTERS
}

\section{Patient-oriented research can be meaningful for clinicians and trialists as well as patients}

We read with interest the CMAJ Analysis by Aubin and colleagues, ${ }^{1}$ which identified issues in developing and measuring the impact of patient-oriented research. We are studying cerebral venous thrombosis (CVT), an uncommon cause of stroke, in a national trial (Study of rivaroxaban in CeREbral venous Thrombosis - SECRET) and parallel registry (TOP-SECRET). By incorporating patient-oriented research through consultation and patient-related outcome measures, we hope our study will be clinically meaningful to patients and providers, and will provide new knowledge on the natural history of outcomes that are impactful to patients who survive.

The standard outcome for stroke trials, the modified Rankin Scale, poorly accounts for "invisible" but life-altering deficits like pain, mood, fatigue and subtle cognitive issues. As functional outcomes improve, with less death and dependence, accounting for invisible outcomes assumes heightened importance. In CVT, functional outcomes are good in $80 \%-90 \%$ of cases, ${ }^{2}$ but more than $60 \%$ have residual invisible symptoms. ${ }^{3}$ Our trial has a patient engagement core to vet our assessment battery and educational materials. We also formally consulted patients who had CVT and their supporters through surveys and an in-person forum. We collaborated with these patients to identify patient-centred outcomes in CVT and are employing validated metrics to measure these. This strategy evaluates clinical and patientcentred effects, and may also serve as a pragmatic method to power a clinical trial for this uncommon condition.

We agree that common terminology and frameworks for patient-oriented research are needed, but clinicians and researchers should not be dissuaded from incorporating measures for patient-oriented outcomes. Including patient partners in research is pragmatic because their experiences can inform selection of both clinically and personally meaningful outcomes.

\section{Thalia S. Field MD MHSc}

Stroke neurologist, associate professor, Vancouver Stroke Program, University of British Columbia, Vancouver, BC

\section{Vanessa Dizonno MSc}

Study manager, Vancouver Stroke Program, University of British Columbia, Vancouver, BC

\section{Sarah S. Park BSC}

Research assistant, Vancouver Stroke Program, University of British Columbia, Vancouver, BC

\section{Michael D. Hill MD MSC}

Stroke neurologist, professor, Hotchkiss Brain Institute, Cumming School of Medicine, University of Calgary, Calgary, Alta.

Cite as: CMAJ 2020 January 27;192: E100. doi: 10.1503/cmaj.74072

\section{References}

1. Aubin D, Hebert M, Eurich D. The importance of measuring the impact of patient-oriented research. CMAJ 2019;191:E860-4.

2. Ferro JM, Canhão P, Stam J, et al. Prognosis of cerebral vein and dural sinus thrombosis. Results of the International Study on Cerebral Vein and Dural Sinus Thrombosis (ISCVT). Stroke 2004;35:664-70.

3. Hiltunen S, Putaala J, Haapaniemi E, et al. Longterm outcome after cerebral venous thrombosis: analysis of functional and vocational outcome, residual symptoms, and adverse events in 161 patients. J Neurol 2016;263:477-84.

Competing interests: Thalia Field is the principal investigator and Michael Hill is the co-principal investigator of the Study of rivaroxaban in CeREbral venous Thrombosis - SECRET trial. Bayer Canada provided in-kind study medication. No other competing interests were declared 